\title{
Copy number variations and expression of MPDZ are prognostic biomarkers for clear cell renal cell carcinoma
}

\author{
Yong-Sheng Huang ${ }^{1, *}$, Wen-Bin Liu ${ }^{1, *}$, Fei Han ${ }^{1}$, Jun-Tang Yang ${ }^{1}$, Xiang-Lin Hao ${ }^{1}$, \\ Hong-Qiang Chen ${ }^{1}$, Xiao Jiang ${ }^{1}$, Li Yinn ${ }^{1}$, Lin Ao ${ }^{1}$, Zhi-Hong Cui ${ }^{1}$, Jia Cao ${ }^{1}$ and Jin-Yi \\ Liu ${ }^{1}$ \\ ${ }^{1}$ Institute of Toxicology, College of Preventive Medicine, Third Military Medical University, Chongqing 400038, PR China \\ *These authors have contributed equally to this work \\ Correspondence to: Jin-Yi Liu, email: jinyiliutmmu@163.com
}

Jia Cao, email: caojia1962@126.com

Keywords: MPDZ, CNV, CCRCC, prognosis

Received: December 15, 2016 Accepted: July 12, $2017 \quad$ Published: August 12, 2017

Copyright: Huang et al. This is an open-access article distributed under the terms of the Creative Commons Attribution License 3.0 (CC BY 3.0), which permits unrestricted use, distribution, and reproduction in any medium, provided the original author and source are credited.

\section{ABSTRACT}

The vital copy number variation (CNV) plays a crucial role in clear cell renal cell carcinoma (CCRCC). MPDZ inhibit cell polarity associate with osmotic pressure response and cancer-related biological processes. In order to clarify the role of the CNV of MPDZ in the progression of CCRCC, we analyzed the CNV and expression of MPDZ and prognosis in CCRCC patients from The Cancer Genome Atlas data portal. Notably, we found that the deletion of MPDZ was the common CNV, which was present in $\mathbf{2 8 . 6 5 \%}$ of ccRCC patients. With the development of tumors, the percentage of MPDZ deletion increased significantly $(19.38 \%$ in stage $I ; 20.00 \%$ in stage II; 40.94\% in stage III; and $45.00 \%$ in stage IV). The deletion of MPDZ significantly increased cCRCC risk $(P=0.0025)$. Low $M P D Z$ expression associated with its deletion was significantly associated with adverse outcomes in ccRCC patients $(P=0.0342)$. Furthermore, immunohistochemical analysis by tissue microarray showed that MPDZ was expressed at lower levels in tumor tissues compared with adjacent tissues $(P<0.01)$. Kaplan-Meier survival curves showed that CCRCC patients with low MPDZ expression had significantly shorter survival than those with high MPDZ expression $(P=0.002)$. These results indicated that low MPDZ expression associated with $C N V$ is a potential biomarker for the prognosis of CCRCC patients.

\section{INTRODUCTION}

Clear cell renal cell carcinoma (ccRCC), the most common and aggressive histologic subtype of RCC [1], is a malignant kidney neoplasm that may be fatal. It is estimated that approximately $55 \%$ of RCC would be fatal within 5 years after traditional treatment [2-4], because patients are often clinically diagnosed in the advanced phase of the disease. Recently, several novelty methods which primarily rely on clinical features [5-8] and several molecular biological markers [9-12] have blossomed and been externally validated to predict the progression of cancer with metastatic disease after surgical treatment.
These morphologic risk criteria are fairly simplistic to facilitate the early diagnosis of ccRCC. Some genes CNVs, especially gene deletions, have been demonstrated to affect the overall clinical outlook of ccRCC and have been used for tumor diagnosis. However, it is still limited for the early prediction of ccRCC diagnosis. Therefore, it is urgent to identify and verify novel tumor molecular markers for early diagnosis, prognosis and therapy of ccRCC.

The human multi-PDZ domain protein (MPDZ/ MUPP1) gene is located on chromosome 9p22-p24 [13]. Its full-length cDNA (EMBL accession number NM_003829) has an overall length of 7722 bp and 
encodes a protein of 2070 amino acids containing thirteen PDZ domains (Supplementary Figure 1A). As a tight junction-associated protein, MPDZ participates in the formation of connections between epithelial cells and endothelial cells, inhibits cell polarity and is closely related to the cell's osmotic pressure response [14-16]. Although the function of $M P D Z$ in tumorigenesis has rarely been reported, previous research found that MPDZ was involved in the carcinogenic effect of viruses as an interaction partner for the coxsackievirus and adenovirus receptor cytoplasmic domain [17]. The expression of $M P D Z$ in breast cancer tissue was substantially lower than that in the normal mucosa and is correlated with cancer progression and aggression [18]. Clinical association analyses indicated that $M P D Z$ deletion is related to poor survival in nasopharyngeal carcinoma [19]. However, the clinical relevance of $M P D Z$ genetic alterations in ccRCC has not been addressed.

Osmotic pressure is an important factor in ccRCC progression. $M P D Z$ is especially significant in the renal osmoadaptive response $[15,20]$. It is reasonable to presume that $M P D Z$ may play an important role in ccRCC. In the present study, we found that the deletion of $M P D Z$ was frequently detected in ccRCC patients and the deletion of $M P D Z$ was negatively correlated with its transcriptional expression. We also found that both the deletion of $M P D Z$ and the expression of $M P D Z$ were significantly associated with poor outcomes in patients with ccRCC. The molecular link between the deletion of $M P D Z$ and cancer-specific outcomes suggests that MPDZ is a potential tumor suppressor gene in ccRCC. It provides a novel tumor molecular marker for diagnosis, prognostic and therapeutic purposes for patients with ccRCC.

\section{RESULTS}

\section{The CNV of MPDZ frequently co-occurs in ccRCC patients from the TCGA cohort}

To evaluate the impact of the CNV of $M P D Z$ on clinical outcomes, we assembled datasets from TCGA. The CNV of MPDZ was frequently detected in ccRCC patients as follows: $28.65 \%$ of deletions and $2.88 \%$ of amplifications (Figure 1A). The same distribution of CNV was performed for total lymph nodes and lymph node status (Figure 1C-1D), but not for gender $(\mathrm{P}=0.0014$; Figure 1B). However, with an increase of clinical stage, the percentage of CNV also significantly increased as follows: $19.38 \%$ of deletions and $2.33 \%$ of amplifications in stage I; $20.00 \%$ of deletions and $3.64 \%$ of amplifications in stage II; $40.94 \%$ of deletions and $3.94 \%$ of amplifications in stage III; and $45.00 \%$ of deletions and $2.50 \%$ of amplifications in stage IV (Figure $1 \mathrm{E})$. The similar distribution of $M P D Z \mathrm{CNV}$ was seen in different Fuhrman nuclear grades (Figure 1F).

\section{The deletion of $M P D Z$ is associated with pathologic features in ccRCC patients from the TCGA cohort}

Because gene deletion may lead to adverse outcomes in ccRCC, we further analyzed the relationship between the deletion of $M P D Z$ and pathologic features. The relationship between the deletion of $M P D Z$ and pathologic features that correlate with adverse outcomes are listed in Table 1. We found that the deletion of $M P D Z$ was significantly correlated with gender $(\mathrm{P}=0.0096)$, Fuhrman nuclear grade $(\mathrm{P}<0.0001)$, AJCC stage $(\mathrm{P}<0.0001)$, pathological T stage $(\mathrm{P}<0.0001)$ and pathological $\mathrm{M}$ stage $(\mathrm{P}=0.0015)$, but not with age, pathological $\mathrm{N}$ stage, total lymph nodes and lymph node status. A subgroup analysis by pathological $\mathrm{T}$ stage revealed that the deletion of $M P D Z$ in T1 was significantly different from T2, T3 and $\mathrm{T} 4(\mathrm{P}<0.05)$, but not in any other two groups.

\section{The deletion of $M P D Z$ is significantly associated with poor outcomes in ccRCC patients from the TCGA cohort}

Due to the deletion of $M P D Z$ was significantly correlated with Fuhrman nuclear grade and AJCC stage, the competing risk model was applied to survival analysis. The deletion of $M P D Z$ is significantly associated with poor outcomes in ccRCC patients $(\mathrm{P}=0.0025$; Figure $2 \mathrm{~A})$. A subgroup analysis by clinical stage and Fuhrman nuclear grade $(\mathrm{G})$ revealed that the deletion of $M P D Z$ was associated with overall survival in stage III-IV $(\mathrm{P}=0.0337$; Figure 2C), G 1-2 ( $\mathrm{P}=0.0385$; Figure $2 \mathrm{D})$ and $\mathrm{G} 3-4$ $(\mathrm{P}=0.0052$; Figure $2 \mathrm{E})$, but not in stage I-II $(\mathrm{P}=0.0903$; Figure $2 \mathrm{~B})$. We also assessed the impact of $M P D Z$ copy number on CSS using UCSC Xena software program. Whether in copy number or copy number with a gene level database, the CNV of $M P D Z$ is significantly associated with worse CSS in patients with ccRCC (Supplementary Figure 2A-2C).

\section{The deletion of $M P D Z$ is negatively correlated with its transcriptional expression from the TCGA cohort}

To determine the association between the deletion and the expression of $M P D Z$, a correlation analysis was performed based on the 502 ccRCC tissue samples with both gene expression and deletion information. The MPDZ transcriptional expression in the low copy number group was significantly lower than that in high copy number group along with changes in the clinical stage and Fuhrman nuclear grade $(\mathrm{G})$, respectively (Figure $3 \mathrm{~A}-3 \mathrm{~B}$ ). We further investigated $M P D Z$ expression levels between the deletion and wild-type group. Consistent with previous results, the expression of $M P D Z$ was significantly downregulated 
in the deletion group compared with the wild-type group $(\mathrm{P}<0.05$, Figure 3C). Furthermore, compared with the wildtype group, the expression of $M P D Z$ in the deletion group was significantly downregulated in stage I, stage IV and G4 $(\mathrm{P}<0.05$; Figure $3 \mathrm{D}-3 \mathrm{E})$, but not in stage II, stage III, G2 and $\mathrm{G} 3$, respectively (Figure 3D-3E).

A

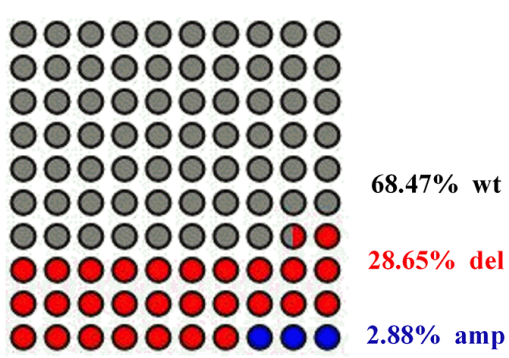

Total $\mathbf{n}=\mathbf{5 2 0}$

C

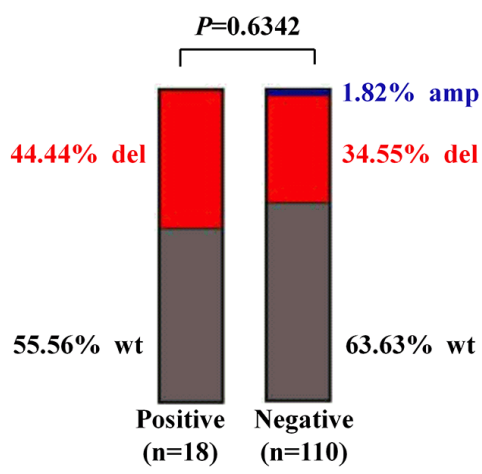

Lymph node status

$\mathbf{E}$

$78.29 \%$ wt $19.38 \%$ del $2.33 \%$ amp

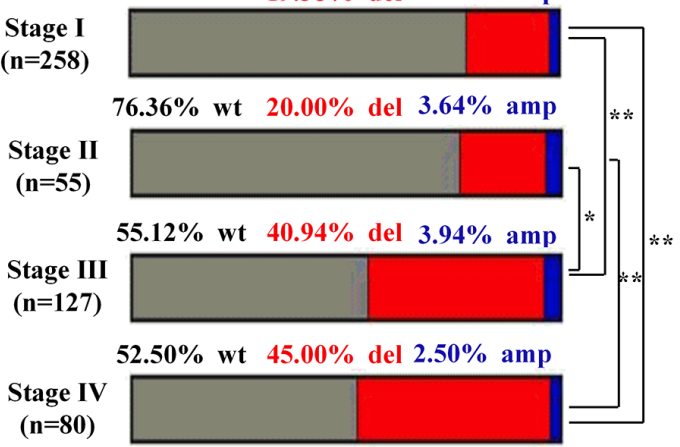

$M P D Z$ expression is significantly downregulated and associated with the poor survival of ccRCC patients from the TCGA cohort

Because the deletion of $M P D Z$ is an important regulation mechanism for its transcriptional expression,

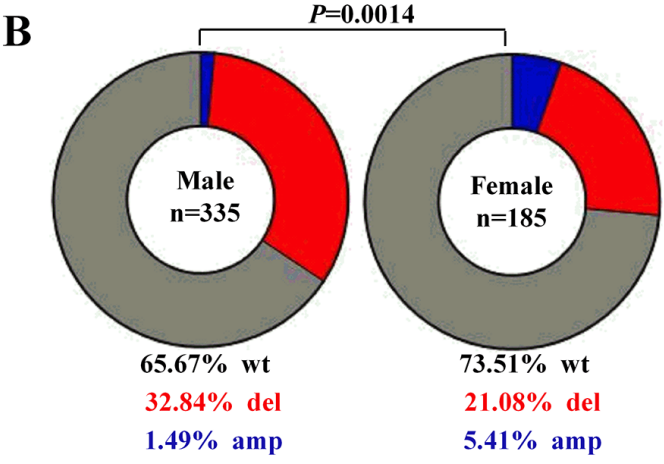

D

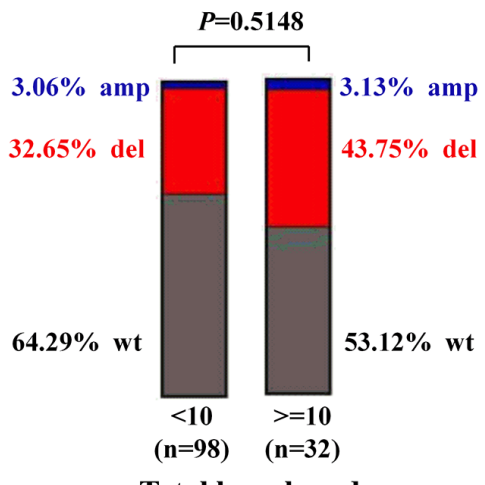

Total lymph nodes

F

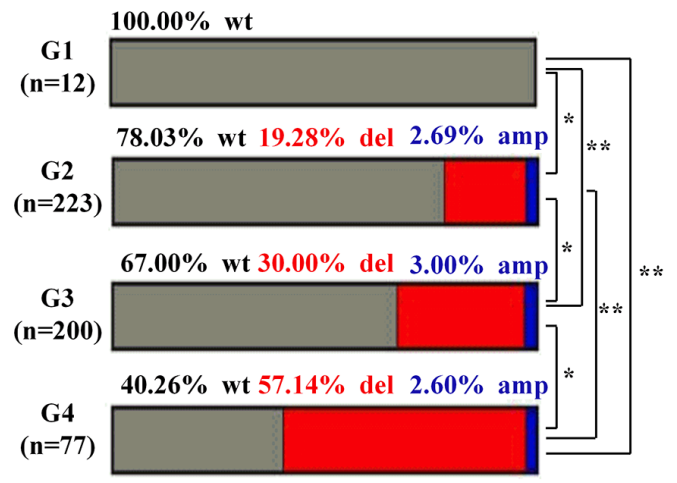

Figure 1: The CNV of MPDZ was frequently detected in ccRCC patients from the TCGA Cohort. (A) The percentage of wild-type, deletions and amplifications in ccRCC, wt, wild-type; del, deletions; amp, amplifications. (B) Different percentage wild-type, deletions and amplifications in genders. (C) Different percentage of wild-type, deletions and amplifications in different lymph node status. (D) Different percentage of wild-type, deletions and amplifications in different total lymph nodes group. (E) Different percentage of wildtype, deletions and amplifications in different clinical stages. (F) Different percentage of wild-type, deletions and amplifications in different Fuhrman nuclear grades. The frequencies of deletions between different groups were used Chi-square test, Multiple comparisons that a $2 \times$ 2 contingency table of expected and observed deletion frequencies were used to analyze more than two groups, with a P-value cut-off set at 0.05 with stringent false discovery rate control (Bonferroni's method). $* \mathrm{P}<0.05, * * \mathrm{P}<0.01$. 
Table 1: Clinical factor and MPDZ copy number in ccRCC patients

\begin{tabular}{|c|c|c|c|c|c|}
\hline \multirow[t]{2}{*}{ Clinical factor } & \multirow[t]{2}{*}{ Numbers } & \multicolumn{2}{|c|}{ MPDZ copy number status } & \multirow[t]{2}{*}{$\chi^{2}$} & \multirow[t]{2}{*}{$P$ value } \\
\hline & & Wt & Deletion & & \\
\hline Total & 505 & 356 & 149 & & \\
\hline Age (year) & & & & 3.52 & 0.0608 \\
\hline$<60$ & 229 & 171 & 58 & & \\
\hline$\geq 60$ & 276 & 185 & 91 & & \\
\hline Gender & & & & 6.71 & 0.0096 \\
\hline male & 330 & 220 & 110 & & \\
\hline female & 175 & 136 & 39 & & \\
\hline Total lymph nodes & & & & 1.33 & 0.2491 \\
\hline$<10$ & 95 & 63 & 32 & & \\
\hline$\geq 10$ & 31 & 17 & 14 & & \\
\hline Unknown & 379 & 276 & 103 & & \\
\hline Lymph node status & & & & 0.23 & 0.6324 \\
\hline Negative & 108 & 70 & 38 & & \\
\hline Positive & 17 & 10 & 7 & & \\
\hline Unknown & 380 & 276 & 104 & & \\
\hline Nuclear grade & & & & 23.51 & $<0.0001$ \\
\hline $1+2$ & 229 & 186 & 43 & & \\
\hline $3+4$ & 269 & 165 & 104 & & \\
\hline other & 7 & 5 & 2 & & \\
\hline AJCC stage & & & & 33.45 & $<0.0001$ \\
\hline $\mathrm{I}+\mathrm{II}$ & 305 & 244 & 61 & & \\
\hline $\mathrm{III}+\mathrm{IV}$ & 200 & 112 & 88 & & \\
\hline \multicolumn{6}{|l|}{ Pathological type } \\
\hline $\mathrm{T}$ (primary tumor) & & & & 37.92 & $<0.0001$ \\
\hline $\mathrm{T} 1$ & 255 & 210 & 45 & & \\
\hline $\mathrm{T} 2$ & 66 & 44 & 22 & & \\
\hline $\mathrm{T} 3$ & 174 & 97 & 77 & & \\
\hline $\mathrm{T} 4$ & 10 & 5 & 5 & & \\
\hline $\mathrm{N}$ (regional lymph nodes) & & & & 1.95 & 0.3782 \\
\hline No & 225 & 164 & 61 & & \\
\hline N1 & 17 & 10 & 7 & & \\
\hline NX & 263 & 182 & 81 & & \\
\hline M (distant metastases) & & & & 13.05 & 0.0015 \\
\hline M0 & 409 & 292 & 117 & & \\
\hline M1 & 73 & 42 & 31 & & \\
\hline $\mathrm{MX}$ & 23 & 22 & 1 & & \\
\hline
\end{tabular}


we further investigated $M P D Z$ expression levels in 72 carcinoma tissues and adjacent tissues. MPDZ expression was significantly downregulated in ccRCC tissues compared with adjacent tissues $(\mathrm{P}<0.01$, Figure 4A). Then, we compared the relationship between the expression of $M P D Z$ and clinical characteristics in 525 patients with the clinical pathologic parameters available.
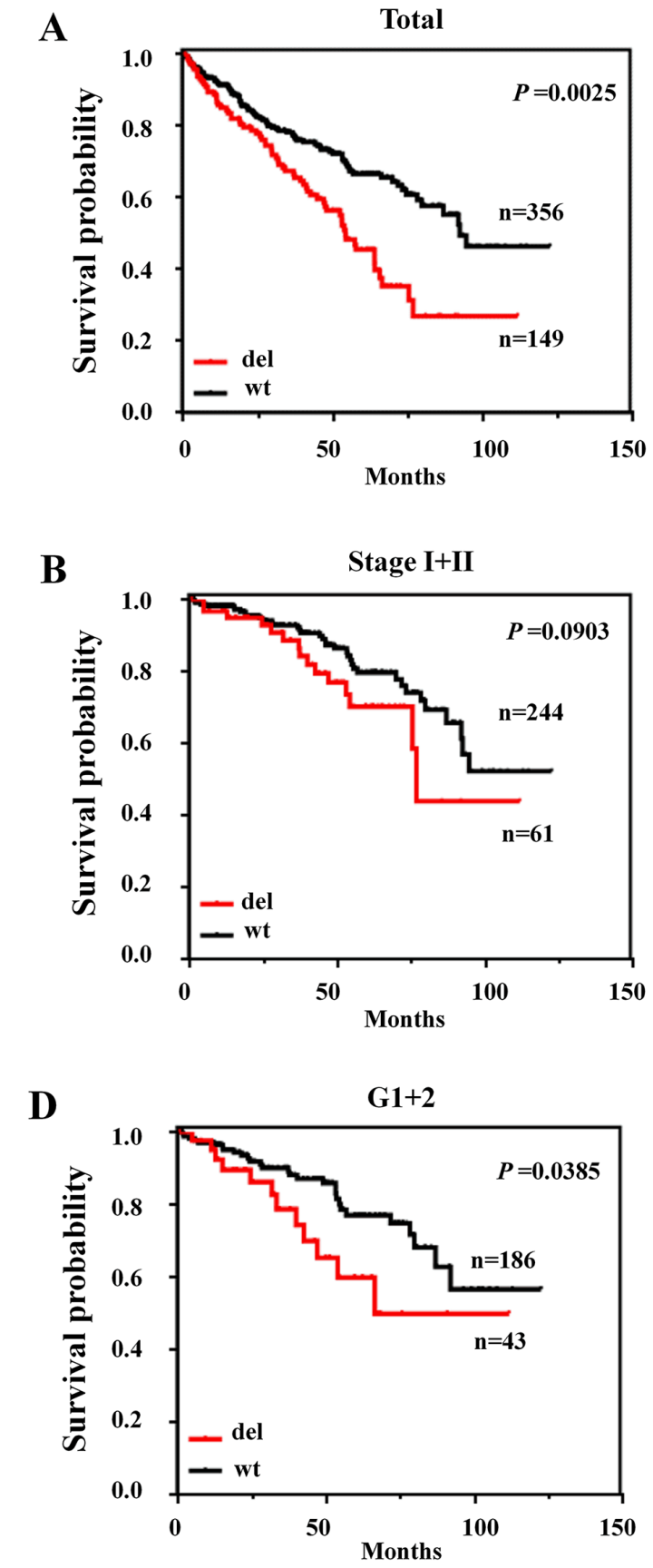

Using median gene expression values as the cutoff, we found that low expression of $M P D Z$ was significantly correlated with Fuhrman nuclear grade $(\mathrm{P}=0.0024)$, AJCC stage $(\mathrm{P}=0.0087)$, pathological $\mathrm{T}$ stage $(\mathrm{P}=0.0078)$, and pathological $\mathrm{M}$ stage $(\mathrm{P}=0.0357)$, but not with age, gender, total lymph nodes, lymph node status and pathological $\mathrm{N}$ stage (Table 2). A subgroup analysis by pathological $\mathrm{T}$
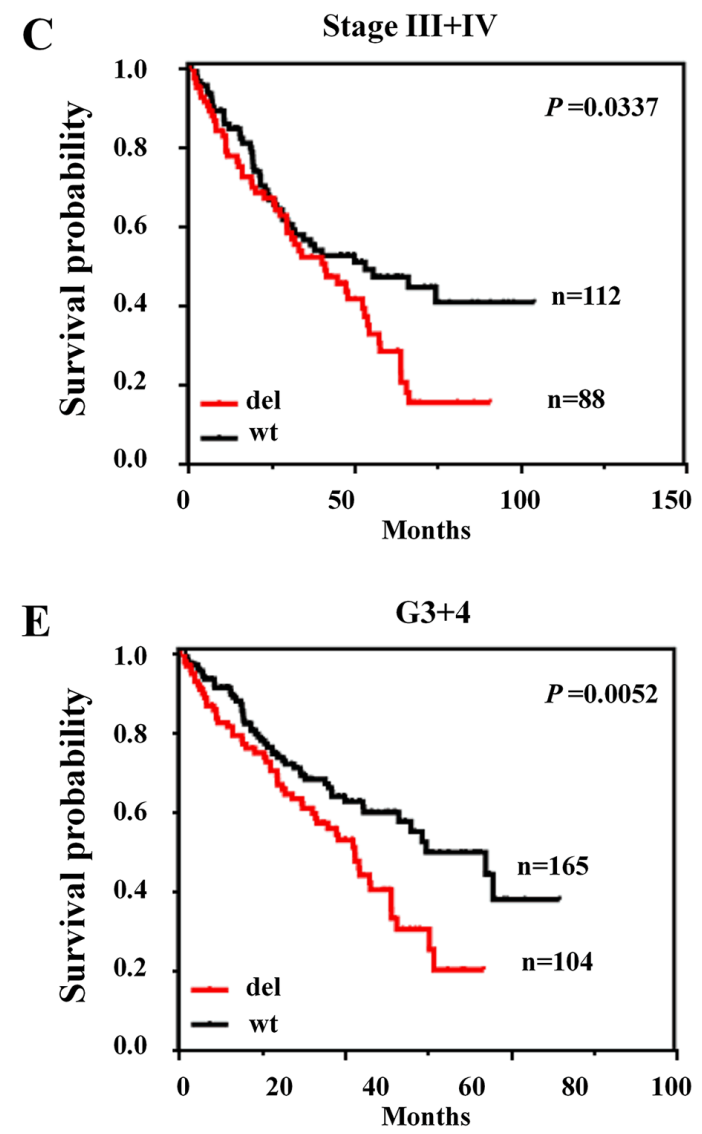

Figure 2: The deletion of MPDZ was associated with poor outcomes in ccRCC from the TCGA Cohort. (A) Kaplan-Meier survival curves show that patients with MPDZ deletions had poorer survival than those with wild-type MPDZ. (B) Kaplan-Meier curves of deletions in patients with AJCC stage I and II. (C) Kaplan-Meier curves of deletions in patients with AJCC stage III and IV. (D) KaplanMeier curves of deletions in patients with Fuhrman nuclear grade 1 and 2. (E) Kaplan-Meier curves of deletions in patients with Fuhrman nuclear grade 3 and 4. 
stage revealed that the low expression of $M P D Z$ in $T 1$ was significantly different from T2, T3 and T4 $(\mathrm{P}<0.05)$, but not in any other two groups.

Moreover, the clinical significance of $M P D Z$ expression in ccRCC patients was assessed by performing a meta-analysis of the association of $M P D Z$ gene expression with outcomes among ccRCC patients.
We observed that the low expression of $M P D Z$ was significantly associated with poor survival in patients with ccRCC ( $\mathrm{P}=0.0342$; Figure 4B). A subgroup analysis clinical stage and Fuhrman nuclear grade $(\mathrm{G})$ revealed that $M P D Z$ was associated with overall survival in stage III-IV ( $\mathrm{P}=0.0331$; Figure 4D) and $\mathrm{G}$ 3-4 $(\mathrm{P}=0.0252$; Figure $4 \mathrm{~F})$, but not in stage I-II $(\mathrm{P}=0.5032$; Figure $4 \mathrm{C})$

A

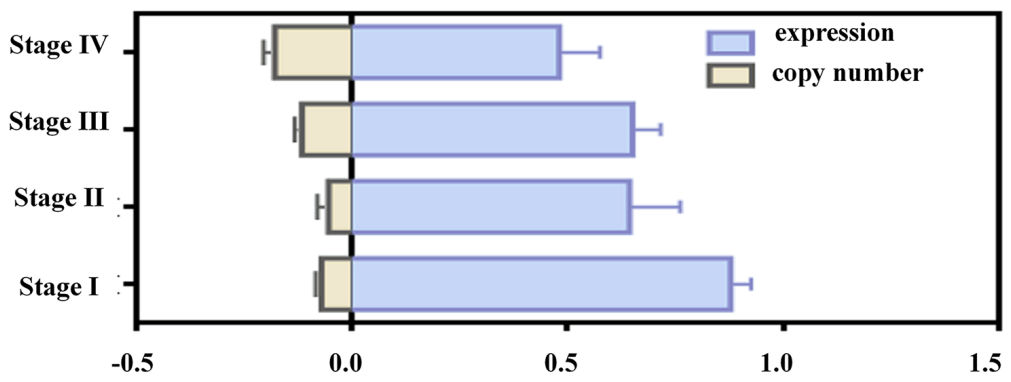

B

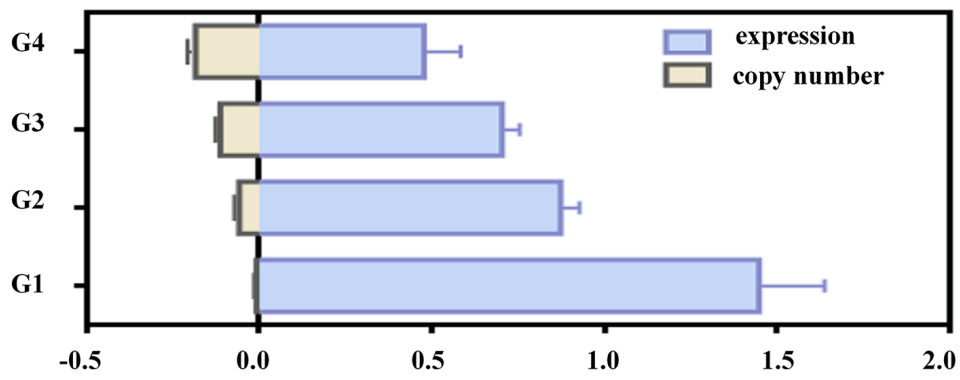

C

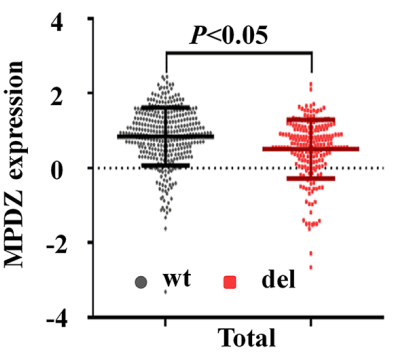

$\mathbf{E}$

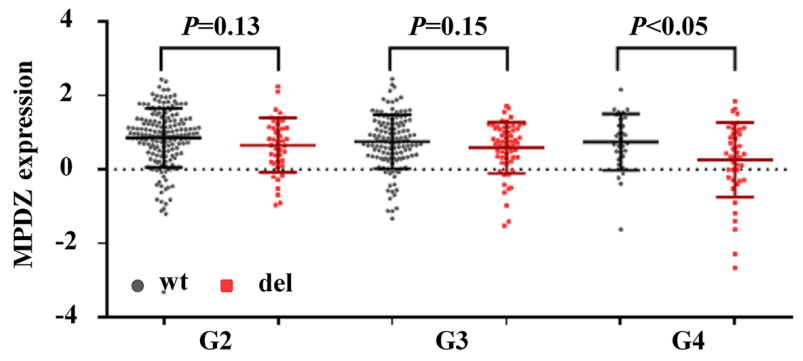

D

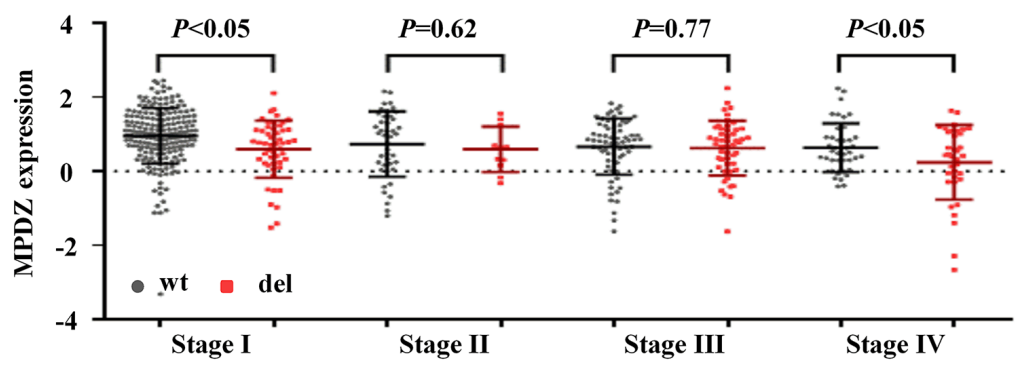

Figure 3: The MPDZ copy number was correlated with mRNA expression level in ccRCC from the TCGA Cohort. The $M P D Z$ mRNA expression and copy number were converted by index. The MPDZ mRNA expression and copy number in different clinical stages (A) and Fuhrman nuclear grades (B). (C) The MPDZ mRNA expression in the wild-type group and deletion group. The MPDZ mRNA expression of wild-type group and deletion group show in different clinical stages (D) and Fuhrman nuclear grades (E). wt, wild type; del, deletion; G, Fuhrman nuclear grades. Two-tailed Wilcoxon test. 
and $\mathrm{G} 1-2(\mathrm{P}=0.5324$; Figure $4 \mathrm{E})$. To further confirm these results, we subsequently performed a meta-analysis using the UCSC Xena software program. Consistent with our results, low $M P D Z$ expression levels are associated with poor overall survival in ccRCC patients (Supplementary Figure 3A-3C).

A

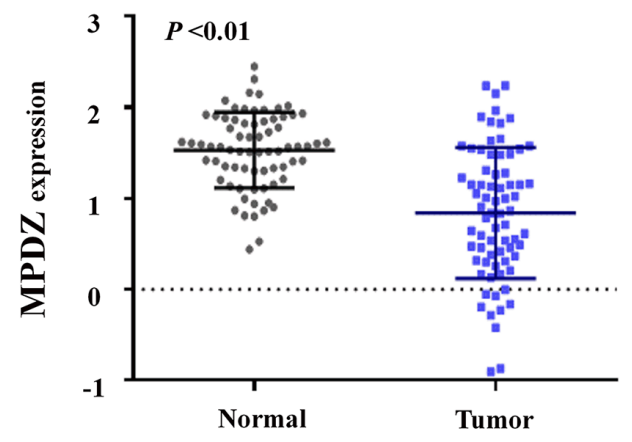

C

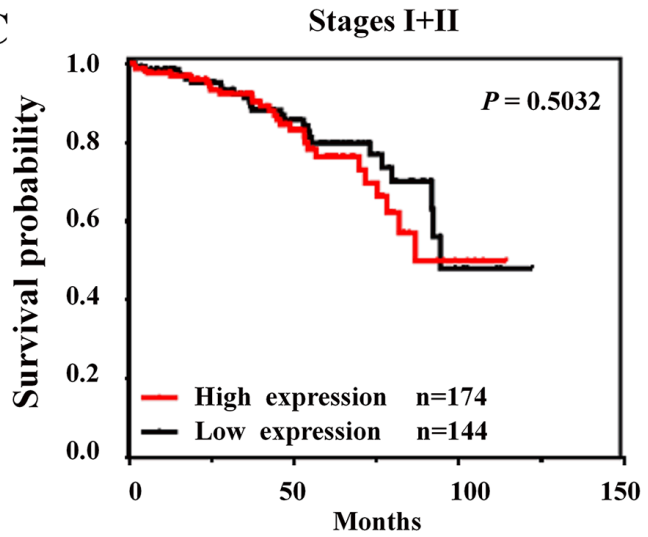

E

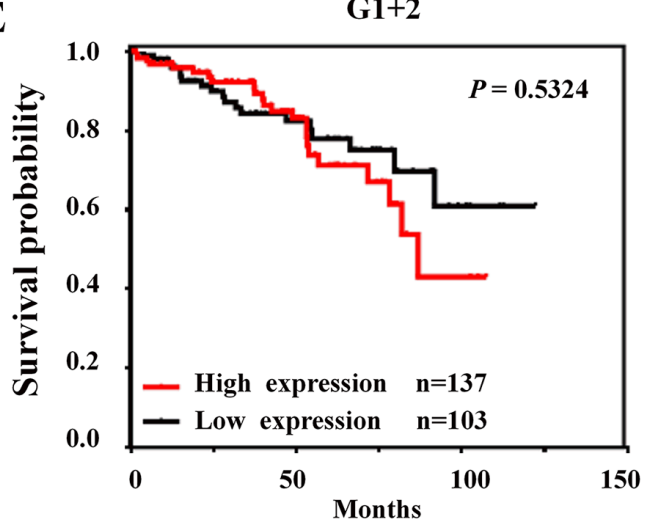

Low MPDZ expression is associated with poor survival of ceRCC patients

To further evaluate the clinical significance of MPDZ, immunohistochemical analysis was performed in a tissue microarray of 150 ccRCC tissues and 30

$\mathbf{B}$

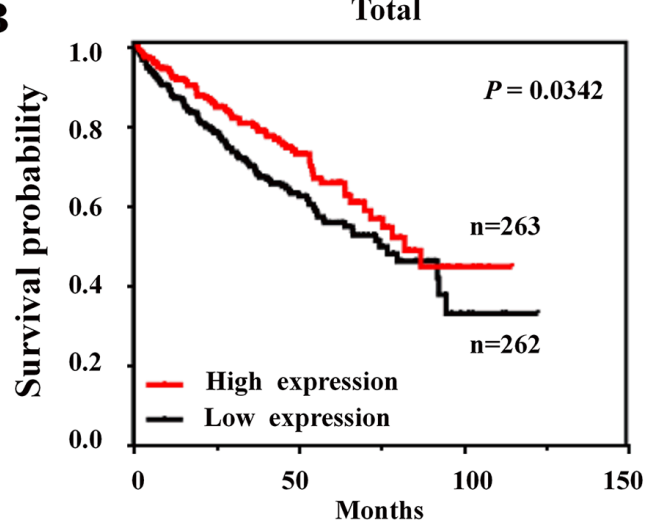

D

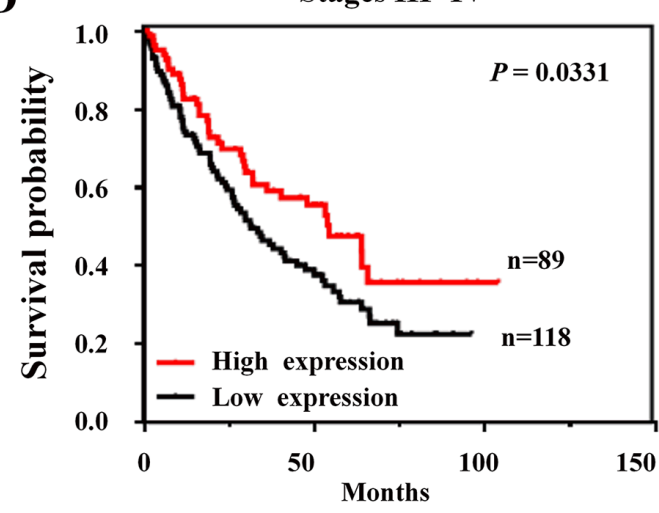

F

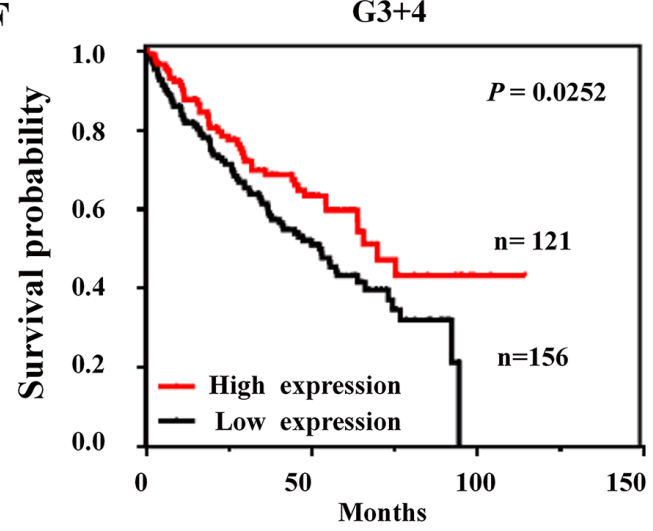

Figure 4: Expression patterns of MPDZ in ccRCC and its clinical significance in the TCGA Cohort. (A) $M P D Z$ expression is significantly downregulated in ccRCC tissues. Two-tailed Wilcoxon test. (B) Kaplan-Meier survival curves showed that patients with low $M P D Z$ expression had poorer survival than those with high $M P D Z$ expression. (C) Kaplan-Meier curves of $M P D Z$ expression in patients with AJCC stage I and II. (D) Kaplan-Meier curves of MPDZ expression in patients with AJCC stage III and IV. (E) Kaplan-Meier curves of $M P D Z$ expression in patients with Fuhrman nuclear grade 1 and 2. (F) Kaplan-Meier curves of MPDZ expression in patients with Fuhrman nuclear grade 3 and 4. 
Table 2: Clinical factor and MPDZ expression in ccRCC patients

\begin{tabular}{|c|c|c|c|c|c|}
\hline \multirow[t]{2}{*}{ Clinical factor } & \multirow[t]{2}{*}{ Numbers } & \multicolumn{2}{|c|}{ MPDZ expression status } & \multirow[t]{2}{*}{$\chi^{2}$} & \multirow[t]{2}{*}{$P$ value } \\
\hline & & High & Low & & \\
\hline Total & 525 & 263 & 262 & & \\
\hline Age (year) & & & & 2.36 & 0.1243 \\
\hline$<60$ & 240 & 129 & 111 & & \\
\hline$\geq 60$ & 285 & 134 & 151 & & \\
\hline Gender & & & & 1.14 & 0.2866 \\
\hline male & 341 & 165 & 176 & & \\
\hline female & 184 & 98 & 86 & & \\
\hline Total lymph nodes & & & & 0.05 & 0.8160 \\
\hline$<10$ & 99 & 41 & 58 & & \\
\hline$\geq 10$ & 32 & 14 & 18 & & \\
\hline Unknown & 394 & 208 & 186 & & \\
\hline Lymph node status & & & & 2.80 & 0.0944 \\
\hline Negative & 111 & 50 & 61 & & \\
\hline Positive & 17 & 4 & 13 & & \\
\hline Unknown & 397 & 209 & 188 & & \\
\hline Nuclear grade & & & & 9.24 & 0.0024 \\
\hline $1+2$ & 240 & 137 & 103 & & \\
\hline $3+4$ & 277 & 121 & 156 & & \\
\hline other & 8 & 5 & 3 & & \\
\hline AJCC stage & & & & 6.89 & 0.0087 \\
\hline $\mathrm{I}+\mathrm{II}$ & 318 & 174 & 144 & & \\
\hline III+IV & 207 & 89 & 118 & & \\
\hline \multicolumn{6}{|l|}{ Pathological type } \\
\hline $\mathrm{T}$ (primary tumor) & & & & 11.90 & 0.0078 \\
\hline $\mathrm{T} 1$ & 267 & 152 & 115 & & \\
\hline $\mathrm{T} 2$ & 68 & 27 & 41 & & \\
\hline $\mathrm{T} 3$ & 179 & 81 & 98 & & \\
\hline $\mathrm{T} 4$ & 11 & 3 & 8 & & \\
\hline $\begin{array}{l}\mathrm{N} \text { (regional lymph } \\
\text { nodes) }\end{array}$ & & & & 3.07 & 0.2159 \\
\hline N0 & 237 & 119 & 118 & & \\
\hline N1 & 17 & 5 & 12 & & \\
\hline NX & 271 & 139 & 132 & & \\
\hline M (distant metastases) & & & & 6.67 & 0.0357 \\
\hline M0 & 421 & 221 & 200 & & \\
\hline M1 & 79 & 29 & 50 & & \\
\hline MX & 25 & 13 & 12 & & \\
\hline
\end{tabular}


A
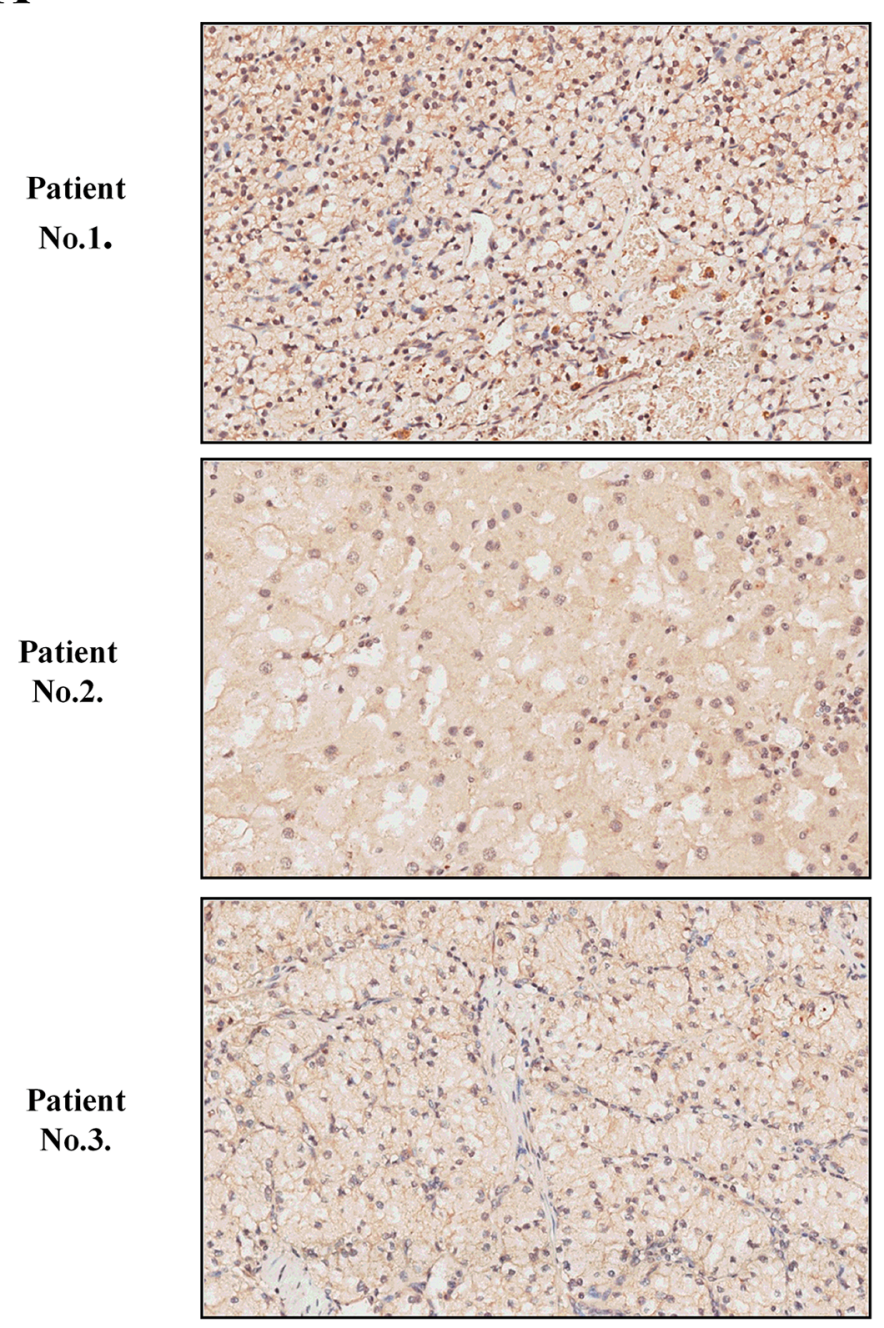
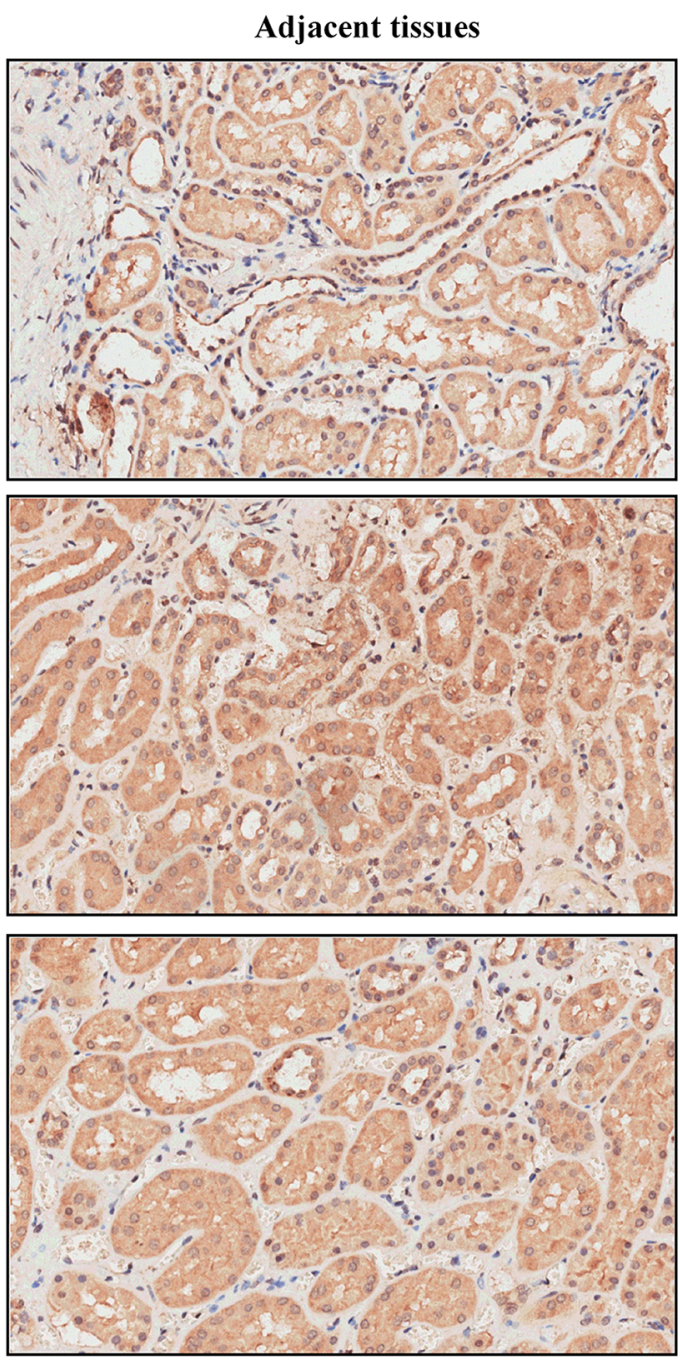

B

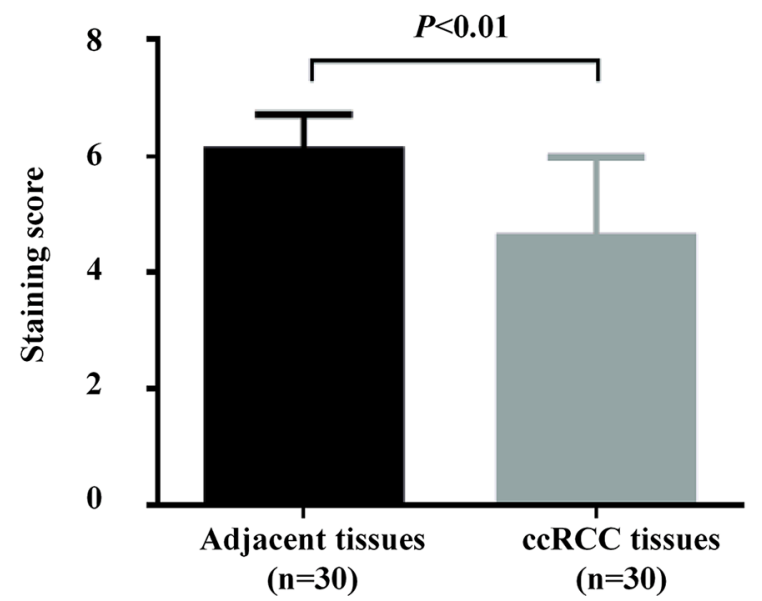

C

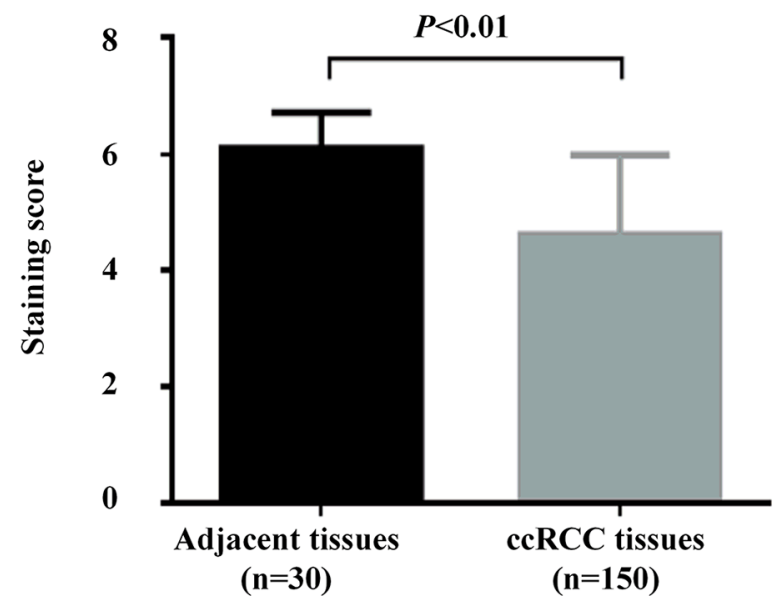

Figure 5: MPDZ expression is significantly downregulated in ccRCC tissues. (A) Immunohistochemistry (IHC) analysis of $M P D Z$ expression levels in adjacent and ccRCC tissues. $M P D Z$ is highly expressed in adjacent tissues. Immunohistological staining assays were performed with an anti-MPDZ antibody (diaminobenzidine (DAB) staining, Magnification, $\times 200$.). (B) $M P D Z$ expression is significantly downregulated in ccRCC tissues according to the paired samples with staining score. (C) $M P D Z$ expression is significantly downregulated in ccRCC tissues according to the total samples with staining score. Two-tailed Wilcoxon test. 
adjacent tissues. We found that MPDZ was expressed at low levels in ccRCC tissues and expressed at high levels in adjacent tissue sample from different patients (Figure 5A). Compared with adjacent tissues, MPDZ expression was significantly downregulated in both paired $(\mathrm{P}<0.01$; Figure 5B) and unpaired $(\mathrm{P}<0.01$; Figure $5 \mathrm{C})$ ccRCC tissues. In order to clarify whether MPDZ protein expression associated with prognosis of ccRCC patients, we used Kaplan-Meier survival curves to determine overall survival in ccRCC patients. Results showed that ccRCC patients with low MPDZ expression had significantly shorter survival than those with high MPDZ expression $(P=0.002$, log-rank test; Figure 6$)$.

\section{DISCUSSION}

Accumulating evidence has demonstrated that predictive and prognostic markers have been proposed to distinguish between poor and favorable risk ccRCC patients $[9,11,12]$. However, these molecular markers stratified the survival curves and discriminated between stage distributions only when they are examined in the progression of ccRCC, but not in the early stage and precancerous lesions. To our knowledge, this is the first analysis on the association between the deletion of $M P D Z$ and outcomes in patients with ccRCC. We found that MPDZ has been frequently deleted and downregulated in ccRCC tissues, which was associated with poor outcomes of ccRCC patients. Due to MPDZ is a protective factor in the renal osmoadaptive response $[15,20]$, the deletion of $M P D Z$ may accelerate the progress of ccRCC under the osmotic pressure. The detection of $M P D Z$ deletion may be a highefficiency predictive method for ccRCC tumorigenesis.

Genetic changes by CNV play an important role in ccRCC and are probably good molecular biomarkers for diagnosis and prognosis of patients [21, 22]. Recently, $\mathrm{CNV}$ silencing of many novel genes that function as putative tumor suppressor genes has been reported to contribute to human cancer $[23,24]$. In this study, we found that $M P D Z$ is a typical CNV gene that commonly showed a CNV status in ccRCC. In addition, we found that there was an unusually simple mutation of $M P D Z$ in ccRCC, which hasn't influence on patients' survival (Supplementary Figure 4A-4C). Because the percentage of deletion has the highest proportion in the $\mathrm{CNV}$ of $M P D Z$, much of the concern among CNV should be focused on the deletion in that the deletion of $M P D Z$ is negatively correlated with its transcriptional expression. These genetic changes suggest that $M P D Z$ is a potential tumor suppressor gene in ccRCC, which provides a novel tumor molecular marker for diagnosis, prognosis and therapy of patients with ccRCC.

In addition, we also observed that the deletion of $M P D Z$ is significantly associated with poor outcomes in patients with ccRCC. However, there are few MPDZ amplifications among patients (only 15 persons from the database), which limits the statistical power because of the small sample. Accounting for the deletion is a common form in the $\mathrm{CNV}$ of $M P D Z$, and the deletion of $M P D Z$ can decrease its expression. Thus, the del vs. wt model
A

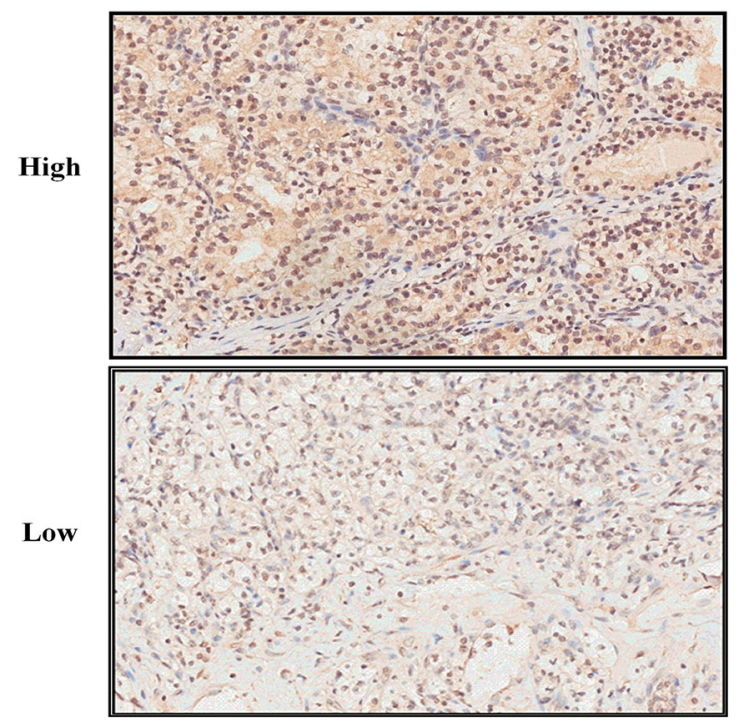

B

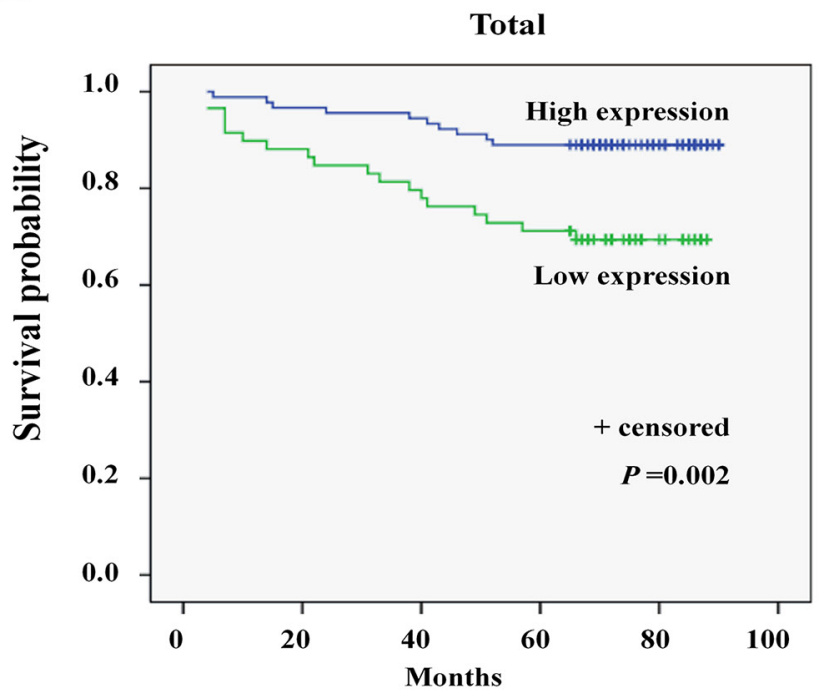

Figure 6: Low expression of MPDZ is significantly associated with poor survival in patients with ccRCC. (A) The different $M P D Z$ expression levels in ccRCC tissues by Immunohistochemistry (IHC) analysis. Immunohistological staining assays were performed with an anti-MPDZ antibody (diaminobenzidine (DAB) staining, Magnification, $\times 200$.). (B) Kaplan-Meier survival curves were shown that patients with low MPDZ expression had poorer survival than those with high MPDZ expression. 
can be considered a conservative estimation of the impact of $M P D Z$ on ccRCC survival. We found that the deletion of $M P D Z$ is associated with poor outcomes in patients with ccRCC. Epigenetic silencing by DNA methylation plays an important role in many novel genes. We further analyzed the state and potential role of $M P D Z$ methylation in ccRCC patients. Interestingly, we found that $M P D Z$ methylation was also associated with poor outcomes in patients with ccRCC (Supplementary Figure 5). It is a potential reason for $M P D Z \mathrm{CNV}$ and expression data did not completely consistent, to some extent. These data are fairly straightforward in offering adequate evidence to confirm the fact that the methylation level of $M P D Z$ is negatively correlated with its transcriptional expression. It is necessary to do further experimental research of the associations between the DNA methylation and the expression of $M P D Z$.

To date, the function of human $M P D Z$ gene with several transcript variants (Supplementary Figure 1B) has not been thoroughly addressed in the literature. Most of studies associated with the function of the MPDZ gene were focused on the cytoskeleton, protein complex formation, signal transduction, cell polarity, cell osmotic pressure reactions and the causes of severe congenital hydrocephalus [14, 15, 25-27]. However, several reports showed that MPDZ participated in nasopharyngeal carcinoma and breast cancer tumorigenesis [17, 18]. However, it should be emphasized that these findings did not show that MPDZ is an independent factor for the prognosis of the tumor. Nevertheless, there is no study on $M P D Z$ gene function in ccRCC. In the present study, we found that $M P D Z$ was frequently deleted and downregulated in ccRCC tissues, which was associated with poor survival of ccRCC patients. Immunohistochemical analysis further certified that MPDZ was expressed at low levels in ccRCC tissues compared with the adjacent tissues. Kaplan-Meier survival curves showed that ccRCC patients with low MPDZ expression had significantly shorter survival than those with high MPDZ expression. These suggested that $M P D Z$ is an independent factor for the prognosis of ccRCC and may play an important role in ccRCC. Interestingly, there had the positive nuclear staining of MPDZ in partly tissue samples particularly in tumor samples. Thus, further experimental research on the associations between MPDZ and ccRCC is needed. We would also study the specific mechanism of MPDZ that has different subcellular localization in cancer in future research.

In summary, our study showed that the genetic silencing of the MPDZ gene by deletions was associated with poor outcomes in patients with ccRCC. It provides knowledge regarding the deletion of $M P D Z$ and expression variations and supports the potential role of prognostic significance, which has important clinical diagnostic and therapeutic implications in ccRCC.

\section{MATERIALS AND METHODS}

\section{Phylogenetic analysis}

MEGA 4 software, which includes neighbor-joining (NJ), Maximum Likelihood (ML) and Bayesian Markov Chain Monte Carlo (MCMC) approaches, was applied to generate an evolutionary tree to explain the phylogenetic relationships of the $M P D Z$ transcript variant $1-\mathrm{X} 16$.

\section{Mutation analysis}

The mutation and its survival analysis were used cBioPortal for Cancer Genomics (http://www.cbioportal. org/), which including Multiregion Sequencing of Clear Cell Renal Cell Carcinoma (IRC, Nat Genet 2014), Clear Cell Renal Cell Carcinoma (U Tokyo, Nat Genet 2013), Kidney Renal Clear Cell Carcinoma (BGI, Nat Genet 2012), Kidney Renal Clear Cell Carcinoma (TCGA, Provisional) and Kidney Renal Clear Cell Carcinoma (TCGA, Nature 2013) studies.

\section{TCGA cohort}

The datasets that included information on gene expression and $\mathrm{CNV}$ in ccRCC patients were downloaded from the TCGA (http://tcgadata.nci.nih.gov). The CNV information of ccRCC patients was acquired from the files entitled "TCGA_KIRC_gistic2thd-2015-02-24" and "TCGA_KIRC_gistic2-2015-02-24." The gene expression data was obtained from the files entitled "TCGA_KIRC_ exp_HiSeqV2_PANCAN-2015-02-24.” After we extracted gene expression information from the genomic data and integrated the clinical and pathologic information through scientific matches, there were 606 tissue samples (534 ccRCC tissues and 72 normal tissues) with $M P D Z$ gene expression information and 525 ccRCC patients with expression, survival and clinical pathologic parameters available. Based on a similar scheme, there were 520 ccRCC patients with $M P D Z$ gene $\mathrm{CNV}$ information and 505 ccRCC patients with the clinical pathologic parameters available were obtained for the deletion of $M P D Z$ and survival analysis. Additionally, we assessed 502 ccRCC patients simultaneously including $M P D Z$ expression and DNA copy number information for correlation analysis. All analyses, including gene expression, survival and CNV incidence, were based on the above database.

\section{CNVs analysis}

After extracted CNVs data, the analysis of CNVs was followed the TCGA publication guidelines (http:// cancergenome.nih.gov/publications). The level $3 \mathrm{CNV}$ of each sample was processed and normalized. The mean copy number estimates of segments overlapping the whole genome were obtained and used for the analysis. Genomic identification of significant targets in cancer (GISTIC) 
algorithm mean cut-offs were used to categorize the gene. The copy number data of each sample were discretized by binning the copy number calls as amplification or deletion using a threshold of $>=1$ for amplification and $<=-1$ for deletion. The formula (number of samples with amplification or deletion in a group)/(total number of samples in a group) were used to calculate the frequency of amplification or deletion.

\section{Tissue microarray (TMA) and immunohistochemistry}

A TMA including 150 ccRCC tissues and 30 adjacent tissues with clinical and prognostic information was obtained from Shanghai Biochip Company Ltd of China. An antibody against MPDZ (SC-135504, Santa Cruz Biotechnology, Santa Cruz, CA, USA) was used to perform immunohistochemical staining in TMA chips as previously described [28]. Two pathologists independently reviewed all core biopsies. The immunostaining was considered positive when $\geq 10 \%$ of the tumour cells was immunoreactive. The intensity of staining was graded semi-quantitatively as negative (scored as 0), weak (1), moderate (2) or strong (3) positivity. The percentage of positive cells, as the extent of immunostaining, was quantified into five groups under microscope: $<10 \%$ positive cells for $0 ; 10-25 \%$ positive cells for $1 ; 26-50 \%$ positive cells for $2 ; 51-75 \%$ positive cells for 3 and $\geq 76 \%$ positive cells for 4. Multiplying the percentage of positive staining and the intensity was used to define expression levels. A final staining score more than six was considered to be high expression.

\section{Kaplan-Meier plotter analysis}

The prognostic value of the MPDZ gene in ccRCC patients was analyzed using the Kaplan-Meier method. The boundaries of high and low expression of $M P D Z$ used the median gene expression value. The competing risk method was applied for CSS analysis. The TCGA datasets to adjust for clinical covariates (AJCC stage I and II vs. III and IV and grade 1 and 2 vs. 3 and 4) used multivariate competing risk models. The classification standards of the deceased patients were according to the previous study [29]. The UCSC Xena (https://genome-cancer.soe.ucsc.edu) was also used to analyze the associations between gene expression, methylation, CNV and survival in ccRCC patients.

\section{Statistical analysis}

The relative standard errors of the mean normalized values were used for gene expression. The gene expression results between the two groups were evaluated using a two-tailed Wilcoxon test and Bonferroni corrected for multiple hypothesis testing. Spearman's rank correlation was applied to analyze the correlation between gene expression and copy numbers. The difference in categorical variables was analyzed by Chi-square test. Multiple comparisons that a $2 \times 2$ contingency table of expected and observed deletion frequencies were used to analyze more than two groups, with a P-value cutoff set at 0.05 with stringent false discovery rate control (Bonferroni's method). A two-sided P-value $<0.05$ was considered statistically significant. Statistical analyses were performed with the SPSS 19.0 software (SPSS, Inc., Chicago, IL, USA).

\section{Abbreviations}

MPDZ, multiple PDZ domain protein; ccRCC, clear cell renal cell carcinoma; $\mathrm{CNV}$, copy number variation; TCGA, the cancer genome atlas.

\section{Author contributions}

YSH and WBL conceived the study, assembled datasets, analyzed data and drafted the manuscript. FH, JTY and XLH, HQC, XJ, LY, LA and ZHC assembled datasets and analyzed data. JC and JYL conceived and supervised the study, analyzed data and finalized the manuscript. All authors were involved in writing the paper and provided final approval of the submitted and published versions.

\section{ACKNOWLEDGMENTS}

This work was supported by grants from the National Natural Science Foundation of China (No. 81573179 and 81573114).

\section{CONFLICTS OF INTEREST}

The authors declare that there are no conflicts of interest.

\section{REFERENCES}

1. Rini BI, Campbell SC, Escudier B. Renal cell carcinoma. Lancet. 2009; 373: 1119-1132.

2. Miller KD, Siegel RL, Lin CC, Mariotto AB, Kramer JL, Rowland JH, Stein KD, Alteri R, Jemal A. Cancer treatment and survivorship statistics, 2016. CA Cancer J Clin. 2016; 66: 271-289.

3. Wagstaff J, Jones R, Hawkins R, Porfiri E, Pickering L, Bahl A, Brown J, Buchan S. Treatment patterns and clinical outcomes in patients with renal cell carcinoma in the UK: insights from the RECCORD registry. Ann Oncol. 2016; 27: 159-165.

4. Pascual D, Borque A. Epidemiology of kidney cancer. Adv Urol. 2008; 2008: 782381. 
5. Purdue MP, Moore LE, Merino MJ, Boffetta P, Colt JS, Schwartz KL, Bencko V, Davis FG, Graubard BI, Janout V, Ruterbusch JJ, Beebe-Dimmer J, Cote ML, et al. An investigation of risk factors for renal cell carcinoma by histologic subtype in two case-control studies. Int J Cancer. 2013; 132: 2640-2647.

6. Frank I, Blute ML, Cheville JC, Lohse CM, Weaver AL, Zincke H. An outcome prediction model for patients with clear cell renal cell carcinoma treated with radical nephrectomy based on tumor stage, size, grade and necrosis: the SSIGN score. J Urol. 2002; 168: 2395-2400.

7. Karakiewicz PI, Briganti A, Chun FK, Trinh QD, Perrotte P, Ficarra V, Cindolo L, De la Taille A, Tostain J, Mulders PF, Salomon L, Zigeuner R, Prayer-Galetti T, et al. Multiinstitutional validation of a new renal cancer-specific survival nomogram. J Clin Oncol. 2007; 25: 1316-1322.

8. Sun M, Shariat SF, Cheng C, Ficarra V, Murai M, Oudard S, Pantuck AJ, Zigeuner R, Karakiewicz PI. Prognostic factors and predictive models in renal cell carcinoma: a contemporary review. Eur Urol. 2011; 60: 644-661.

9. Kim WJ, Gersey Z, Daaka Y. Rap1GAP regulates renal cell carcinoma invasion. Cancer Lett. 2012; 320: 65-71.

10. Xia Y, Liu L, Bai Q, Wang J, Xi W, Qu Y, Xiong Y, Long $\mathrm{Q}, \mathrm{Xu}$ J, Guo J. Dectin-1 predicts adverse postoperative prognosis of patients with clear cell renal cell carcinoma. Sci Rep. 2016; 6: 32657.

11. Fu Q, Chang Y, An H, Fu H, Zhu Y, Xu L, Zhang W, Xu J. Prognostic value of interleukin-6 and interleukin-6 receptor in organ-confined clear-cell renal cell carcinoma: a 5-year conditional cancer-specific survival analysis. Br J Cancer. 2015; 113: 1581-1589.

12. Maroto P, Rini B. Molecular biomarkers in advanced renal cell carcinoma. Clin Cancer Res. 2014; 20: 2060-2071.

13. Ullmer C, Schmuck K, Figge A, Lubbert H. Cloning and characterization of MUPP1, a novel PDZ domain protein. FEBS Lett. 1998; 424: 63-68.

14. Assemat E, Crost E, Ponserre M, Wijnholds J, Le Bivic A, Massey-Harroche D. The multi-PDZ domain protein-1 (MUPP-1) expression regulates cellular levels of the PALS-1/PATJ polarity complex. Exp Cell Res. 2013; 319: 2514-2525.

15. Lanaspa MA, Almeida NE, Andres-Hernando A, Rivard CJ, Capasso JM, Berl T. The tight junction protein, MUPP1, is up-regulated by hypertonicity and is important in the osmotic stress response in kidney cells. Proc Natl Acad Sci U S A. 2007; 104: 13672-13677.

16. Ebnet K, Suzuki A, Ohno S, Vestweber D. Junctional adhesion molecules (JAMs): more molecules with dual functions? J Cell Sci. 2004; 117: 19-29.

17. Coyne CB, Voelker T, Pichla SL, Bergelson JM. The coxsackievirus and adenovirus receptor interacts with the multi-PDZ domain protein-1 (MUPP-1) within the tight junction. J Biol Chem. 2004; 279: 48079-48084.
18. Martin TA, Watkins G, Mansel RE, Jiang WG. Loss of tight junction plaque molecules in breast cancer tissues is associated with a poor prognosis in patients with breast cancer. Eur J Cancer. 2004; 40: 2717-2725.

19. Sheu JJ, Lee CH, Ko JY, Tsao GS, Wu CC, Fang CY, Tsai FJ, Hua CH, Chen CL, Chen JY. Chromosome 3p12.3-p14.2 and 3q26.2-q26.32 are genomic markers for prognosis of advanced nasopharyngeal carcinoma. Cancer Epidemiol Biomarkers Prev. 2009; 18: 2709-2716.

20. Lanaspa MA, Andres-Hernando A, Rivard CJ, Dai Y, Berl T. Hypertonic stress increases claudin-4 expression and tight junction integrity in association with MUPP1 in IMCD3 cells. Proc Natl Acad Sci U S A. 2008; 105: 15797-15802.

21. Becket E, Chopra S, Duymich CE, Lin JJ, You JS, Pandiyan K, Nichols PW, Siegmund KD, Charlet J, Weisenberger DJ, Jones PA, Liang G. Identification of DNA methylationindependent epigenetic events underlying clear cell renal cell carcinoma. Cancer Res. 2016; 76: 1954-1964.

22. Kwiatkowski DJ, Choueiri TK, Fay AP, Rini BI, Thorner AR, de Velasco G, Tyburczy ME, Hamieh L, Albiges L, Agarwal N, Ho TH, Song J, Pignon JC, et al. Mutations in TSC1, TSC2, and MTOR are associated with response to rapalogs in patients with metastatic renal cell carcinoma. Clin Cancer Res. 2016; 22: 2445-2452.

23. Kovac M, Navas C, Horswell S, Salm M, Bardella C, Rowan A, Stares M, Castro-Giner F, Fisher R, de Bruin EC, Kovacova M, Gorman M, Makino S, et al. Recurrent chromosomal gains and heterogeneous driver mutations characterise papillary renal cancer evolution. Nat Commun. 2015; 6: 6336.

24. Jedrak J, Ochab-Marcinek A. Influence of gene copy number on self-regulated gene expression. J Theor Biol. 2016; 408: 222-236.

25. Fujita E, Tanabe Y, Imhof BA, Momoi MY, Momoi T. A complex of synaptic adhesion molecule CADM1, a molecule related to autism spectrum disorder, with MUPP1 in the cerebellum. J Neurochem. 2012; 123: 886-894.

26. Katoh M. Function and cancer genomics of FAT family genes (review). Int J Oncol. 2012; 41: 1913-1918.

27. Balasubramanian S, Fam SR, Hall RA. GABAB receptor association with the PDZ scaffold Mupp1 alters receptor stability and function. J Biol Chem. 2007; 282: 4162-4171.

28. Liu WB, Han F, Jiang X, Chen HQ, Zhao H, Liu Y, Li YH, Huang C, Cao J, Liu JY. TMEM196 acts as a novel functional tumour suppressor inactivated by DNA methylation and is a potential prognostic biomarker in lung cancer. Oncotarget. 2015; 6: 21225-21239. https://doi. org/10.18632/oncotarget.4237.

29. Hakimi AA, Ostrovnaya I, Reva B, Schultz N, Chen YB, Gonen M, Liu H, Takeda S, Voss MH, Tickoo SK, Reuter VE, Russo P, Cheng EH, et al. Adverse outcomes in clear cell renal cell carcinoma with mutations of 3 p21 epigenetic regulators BAP1 and SETD2: a report by MSKCC and the KIRC TCGA research network. Clin Cancer Res. 2013; 19: 3259-3267. 